\title{
Laranja-da-terra: \\ fruta cítrica potencial para o Piauí
}

\author{
Laranja-da-terra: \\ potential citric fruit for Piauí State
}

\author{
Gabriel Barbosa da Silva Júnior ${ }^{1}$; Leonardo Fonseca da Rocha ${ }^{1}$; \\ Francisco Hélcio Canuto Amaral ${ }^{1}$; Marcelo Luiz de Andrade (in memorian) ${ }^{2}$; \\ Raimundo Falcão Neto ${ }^{2}$; Ítalo Herbert Lucena Cavalcante ${ }^{3 *}$
}

\section{Resumo}

O trabalho teve como objetivo a caracterização físico-química de frutos de laranja-da-terra (Ctrius aurantium L). Cinco amostras de dez frutos cada foram colhidas em plantas de laranja-da-terra produzidos em pomares domésticos do município de Bom Jesus/PI e caracterizadas pela avaliação de diâmetro e altura, número de sementes por fruto, massa de frutos sólidos solúveis (SS), acidez titulavel (AT), vitamina C e relação SS/AT. A laranja-da-terra tem boas qualidades físico-quimicas e é uma fonte natural de vitamina $\mathrm{C}$ com media de $125,76 \mathrm{mg} / 100 \mathrm{~g}$ de fruta fresca, com acidez baixa e elevados sólidos solúveis e "ratio".

Palavras-chave: Fruta cítrica, qualidade de fruto, Citrius aurantium L.

\begin{abstract}
The work had as objecive the phisico-chemical caracterization of laranja-da-terra fruits. Five samples of 10 fruits were harvested in laranja-da-terra plants produced in home orchards of the municipality of Bom Jesus/PI and caracterized by evaluation of lenght and width, number of the seeds per fruits, weigth, soluble solids (SS), titulable acidity (TA), vitamin C and SS/AT ratio. Laranja-da-terra has good quality phisico-chemical and is a natural source of vitamin C with an average of $125.76 \mathrm{mg} / 100 \mathrm{~g}$ of fresh fruit, low titratable acidity and high values of soluble solids and SS/AT ratio.
\end{abstract}

Key words: Citric fruit, fruit quality, Citrus aurantium L.

\footnotetext{
${ }^{1}$ Estudante de Engenharia Agronômica, CPCE / UFPI - Universidade Federal de Piauí, Bom Jesus, PI. Bolsista PIBIC/ CNPq. E-mail: gabrielbarbosa@ufpi.br

${ }^{2}$ Mestrando em Solos e Nutrição de Plantas, CPCE/UFPI - Universidade Federal de Piauí, Bom Jesus, PI. E-mail: falcao@ufpi. br

${ }^{3}$ Prof., Dr., CPCE/UFPI - Universidade Federal de Piauí. Bom Jesus, PI. E-mail: italohlc@ufpi.edu.br

* Autor para correspondência
} 


\section{Introdução}

A citricultura é um relevante segmento da economia mundial, presente em mais de 80 países com uma produção atual de mais de 60 milhões de toneladas. Atualmente o Brasil é o maior produtor de laranja do mundo, seguido por Estados Unidos da América, México, Índia e Espanha (FAO, 2008). No Brasil, a citricultura encontra-se concentrada em poucas variedades de laranja (Hamlin, Natal, Pêra e Valência) o que concentra a produção em parte do ano e aumenta o risco de incidência generalizada de pragas e doenças. Entretanto, ainda há espécies cítricas sub-utilizadas seja como porta-enxerto (sapota branca), (CAVALCANTE et al., 2008), seja para a produção de frutos de mesa, como a laranja da terra.

A laranja-da-terra (Citrus aurantium L.), pertencente à família Rutaceae, é popularmente conhecida como laranja-azeda tem origem no Sudoeste asiático e no Brasil é encontrada em pomares domésticos. Sua multiplicação é feita através de sementes e as plantas são caracterizadas por apresentarem porte mediano, flores aromáticas, espinhos agudos e frutos do tipo baga, arredondado com casca medianamente grossa e amarga, sendo utilizada na alimentação, medicina e indústria (LORENZI et al., 2006). No Piauí apresenta produção no segundo semestre do ano.

A aparência de frutos é caracterizada pelo tamanho, forma, cor, condições e ausência de desordens mecânicas, fisiológicas e patológicas (KAYS, 1999), tanto externa quanto internamente. A aparência externa é o primeiro critério utilizado pelo consumidor no julgamento da qualidade dos frutos (KAYS, 1999), no entanto, outros atributos de qualidade como sabor, aroma, textura, valor nutritivo e segurança alimentar, devem ser considerados na compra do produto (CHITARRA; CHITARRA, 1990). Os componentes responsáveis pela qualidade nutricional dos produtos são vitaminas, minerais, açúcares solúveis, amido, fibras, hemiceluloses e lignina (KAYS, 1999).
Adicionalmente, algumas substâncias químicas que condicionam valor nutritivo ao produto frutícola são também responsáveis pelo sabor, como é o caso dos sólidos solúveis, açúcares e ácidos orgânicos (MATTHEIS; FELLMAN, 1999).

Os frutos da laranja-da-terra são consumidos in natura na forma de sucos e temperos e a casca utilizada na culinária para a produção de doces e geléias (LORENZI et al., 2006). Entretanto, os trabalhos que têm como objeto de estudo essa potencial frutífera plenamente adaptada às condições do Piauí são escassos na literatura, o que determina a importância da pesquisa direcionada inicialmente para a caracterização do produto.

O objetivo do presente trabalho foi realizar a caracterização físico-química de frutos de laranjada-terra.

\section{Material e Métodos}

O presente experimento foi realizado no Laboratório de Físico-química da Universidade Federal do Piauí, Campus Professora Cinobelina Elvas, Bom Jesus-PI, em outubro de 2007.

O local apresenta precipitação pluviométrica media de 900 a $1200 \mathrm{~mm} /$ ano e temperatura media de $26,5{ }^{\circ} \mathrm{C}$, embora durante o ano seja comum a temperatura de $40{ }^{\circ} \mathrm{C}$ (VIANA et al., 2002).

Foram utilizados frutos de Laranja-da-terra (Citrus aurantium L.) produzidos em pomares domésticos do município de Bom Jesus/PI, sem tratos culturais sistemáticos como poda, irrigação, controle de plantas invasoras e fertilização.

Os frutos foram colhidos manualmente à completa maturação de acordo com a homogênea coloração externa, devidamente acondicionados em sacolas de papel de maneira a evitar escoriações e imediatamente conduzidos ao laboratório, onde foram selecionados, uniformizados quanto ao tamanho e submetidos à caracterização físicoquímica. 
Avaliaram-se cinco repetições de dez frutos cada uma, a partir de análises físicas e químicas. As análises físicas compreenderam: i) diâmetros longitudinal e transversal: determinados com paquímetro (precisão 0,01) e expressos em $\mathrm{mm}$ e, de posse desses resultados, foi obtido o formato do fruto pela relação DL/DT; ii) massa dos frutos: registrada por gavimetria usando-se balança analítica (precisão de 0,01 g) e expressa em g; número de sementes: determinada a partir de contagem manual. As análises químicas (Metodologia descrita por (LARA et al., 1976) compreenderam: i) acidez titulável: foi tomada $20 \mathrm{~g}$ de polpa de laranja-daterra, adicionando-se água destilada até completar um volume de $100 \mathrm{~mL}$. Em seguida tomou-se uma amostra da mesma $(20 \mathrm{~mL})$ e adicionou-se quatro gotas do indicador fenolftaleína. Esta suspensão foi titulada com uma solução de $0,1 \mathrm{NaOH}$, com resultados expressos em porcentagem; ii) vitamina $\mathrm{C}$ : foi tomado $5 \mathrm{~g}$ de polpa de laranja-da-terra, adicionada água destilada até completar um volume de $100 \mathrm{~mL}$ e agregado $1 \mathrm{~mL}$ de solução de amido (1\% de concentração). Tomou-se uma alicota de $20 \mathrm{~mL}$ da mesma ao qual foi titulada com Iodo $(1 \mathrm{~N})$, com resultados expressos em $\mathrm{mg} / 100 \mathrm{~g}$ de fruta fresca; iii) sólidos Solúveis (SS): SS foram determinados em refratômetro digital Abbe não termocompensado e expressos em Brix; iv) pH: Alícota com leitura em peagômetro; v) foram registrados também os valores médios do "Ratio" a partir da proporção SS/AT.

Os valores de média e desvio padrão das amostras foram calculados com base nas recomendações de Ferreira (2000) usando o software ASSISTAT (SILVA; AZEVEDO, 2006).

\section{Resultados e Discussão}

De uma forma geral, a laranja-da-terra apresenta potencial para consumo de fruto in natura (Tabela 1). A massa de frutos apresentou valores variando de $231,785 \mathrm{~g}$ a 285,185g. Comparativamente às médias de Cavalcante, Martins e Stuchi (2006), em estudo realizado na região de Bebedouro com dezoito variedades de laranja, observa-se que os resultados do presente trabalho são maiores, i.e. frutos de maior massa, demonstrando que os frutos de laranjada-terra, quanto à essa variável, são passíveis de consumo in natura, como uma nova alternativa para produtores e potenciais consumidores no Piauí.

Tabela 1. Características físicas [massa (MF), número de sementes (NS), diâmetros longitudinal (DL) e transversal (DT) e relação DL/DT] de frutos de laranja-da-terra. Bom Jesus - PI, 2008.

\begin{tabular}{|c|c|c|c|c|c|}
\hline \multirow{2}{*}{ REPETIÇÃO } & MF & \multirow[t]{2}{*}{ NS } & \multirow[t]{2}{*}{$\mathrm{DL}$} & DT & \multirow[t]{2}{*}{$\mathrm{DL} / \mathrm{DT}$} \\
\hline & $-\mathrm{g}-$ & & & $\mathrm{cm}$ & \\
\hline 1 & 285,185 & 26 & 8,10 & 8,45 & 0,96 \\
\hline 2 & 277,665 & 32 & 8,12 & 8,20 & 0,99 \\
\hline 3 & 245,325 & 14,5 & 7,92 & 8,32 & 0,95 \\
\hline 4 & 281,46 & 23,5 & 8,05 & 8,00 & 1,01 \\
\hline 5 & 231,785 & 22 & 8,85 & 8,12 & 1,09 \\
\hline Média & 264,284 & 23,6 & 8,210 & 8,220 & 1,00 \\
\hline DP & 26,922 & 7,321 & 0,602 & 0,282 & 0,056 \\
\hline
\end{tabular}

$\mathrm{DP}=$ desvio padrão 
Os valores dos diâmetros longitudinal e transversal estão contidos na Tabela 1 . O diâmetro longitudinal apresentou uma variação aproximada de $10 \%$, com valores médios não superiores a $8,85 \mathrm{~cm}$. Quanto ao diâmetro transversal, os resultados médios oscilaram entre 8,00 e $8,45 \mathrm{~cm}$, valores que conferem ao fruto da laranja-da-terra, de acordo com parâmetro estabelecido por Viégas (1991), a classificação como "grandes" e enfatizam o potencial para consumo como fruto de mesa.

A partir da razão entre os dois diâmetros estudados, obtém-se o valor DL/DT, importante para determinar o formato do fruto, uma característica relevante para a comercialização do produto como fruto de mesa. Quanto a essa variável, os valores variaram de 0,95 a 1,09 , com média geral equivalente a 1,0, isto é, os frutos são de formato arredondado e possuem diâmetro longitudinal semelhante ao transversal, classificando-o como fruto de qualidade para mesa.

Por outro lado, uma desvantagem dos frutos oriundos dessa frutífera cítrica é o elevado número de sementes (em média 23,6 sementes/fruto), característica indesejável a frutos de mesa, mas que pode ser objeto de futuros trabalhos de melhoramento vegetal. A quantidade de sementes registrada para a laranja-da-terra é alta quando comparadas a outras frutas cítricas, como a laranjeira lima que apresenta de 5 a 6 sementes/fruto (GOMES, 2007). Em complemento, essa é uma característica interessante para as espécies indicadas como porta-enxerto cítrico, como se observa nos valores semelhantes reportados por Soares Filho et al. (2002), que registraram 26,3 sementes na laranjeira Azeda Comum, 21,2 no limoeiro Volkameriano, 16,6 na tangerineira Clementina, 13,8 na laranjeira Azeda Double Calice, 7,6 no limoeiro Cravo e 5,7 na tangerineira Sunki.

Dentre as variáveis químicas (Tabela 2), os sólidos solúveis (expressos em Brix) apresentaram valores médios variando entre 13,1 e 15,5 Brix, portanto elevados se comparados com os obtidos por Cavalcante, Martins e Stuchi (2006), que registrou o mais elevado SS equivalente 13,6 Brix para a variedade Hamlin, uma das quatro variedades de laranja mais plantadas no Estado de São Paulo. Os sólidos solúveis são compostos solúveis em água e importantes na determinação da qualidade da fruta (KLUGE et al., 2002).

Para a acidez titulável (AT) os valores observados para a laranja-da-terra encontram-se abaixo dos apresentados, por exemplo, para a variedade Hamlin (CAVALCANTE; MARTINS; STUCHI, 2006). O consumo da maioria das laranjeiras depende do teor de diminuição do teor de acidez até um ponto em que seu suco se torne agradável ao paladar (AWAD, 1993), ponto a partir do qual a diminuição da AT passa a representar um fator deletério da qualidade do fruto.

Tabela 2. Características químicas [pH, sólidos solúveis (SS), acidez titulável (AT), "Ratio" e vitamina C (Vit-C)] de frutos de laranja-da-terra. Bom Jesus - PI, 2008.

\begin{tabular}{lccccc}
\hline \multirow{2}{*}{ Repetição } & $\mathrm{pH}$ & $\begin{array}{c}\text { SS } \\
\text { - Brix }\end{array}$ & $\begin{array}{c}\text { AT } \\
\%\end{array}$ & $\begin{array}{c}\text { "Ratio" } \\
\text { SS/AT }\end{array}$ & $\begin{array}{c}\text { Vit. C } \\
\text { mg/100g de polpa }\end{array}$ \\
\hline 1 & 5,43 & 15,5 & 0,18 & 86,11 & 115,15 \\
2 & 5,27 & 15,2 & 0,25 & 60,80 & 113,63 \\
3 & 5,36 & 14,7 & 0,23 & 63,91 & 130,93 \\
4 & 5,28 & 15,1 & 0,24 & 62,91 & 108,42 \\
5 & 5,32 & 13,1 & 0,21 & 62,38 & 143,11 \\
\hdashline Média & 5,337 & 14,740 & 0,227 & 67,22 & 122,260 \\
DP & 0,080 & 0,943 & 0,039 & 10,618 & 17,171 \\
\hline
\end{tabular}

$\mathrm{DP}=$ desvio padrão 
Para frutos de laranja produzidos com finalidade industrial, foram determinados por Steger (1990) limites inferiores e superiores de acidez titulável que o fruto deve apresentar no momento da colheita, equivalentes a 0,75 e $1 \%$ respectivamente. Nesse sentido, a laranja-da-terra produz frutos de baixa acidez (média máxima de $0,25 \%$, Tabela 2), inclusive com média inferior à "João Nunes", variedade que reconhecidamente é caracterizada por possuir frutos de baixa acidez, com percentuais não superiores a 0,5\% (DONADIO; FIGUEIREDO, 1995).

Quanto ao "ratio" (SS/AT), as médias variaram entre 62,38 e 86,11 , portanto encontram-se bem acima dos apresentados por Cavalcante, Martins e Stuchi (2006) para algumas cultivares tradicionais de laranja cultivadas no Estado de São Paulo como João Nunes, Hamlin e Westin com 23,46; 18,98 e 19,32 respectivamente.

O "ratio" ou relação dos açucares/ácidos inorgânicos é uma relação que apresenta relevância diferenciada para o fruto cítrico se comparada a outras frutas, pois reflete o índice de maturidade do fruto cítrico a partir do balanço do sabor doce: ácido. Conforme Marchi (1993), a faixa de ratio de 12 a 13 é a preferida para a indústria iniciar o processamento; Steger (1990) relacionou o "ratio" entre 12 e 14 como o de melhor sabor para o consumo na forma de suco.

Embora também conhecida como laranja-azeda e com características morfológicas semelhantes de acordo com Lorenzi et al. (2006), a laranja-da-terra oriunda do Piauí avaliada no presente estudo, não apresentou baixos valores acidez, o que caracteriza o sabor azedo.

O conteúdo de vitamina C variou de 108,42 a $143,11 \mathrm{mg} / 100 \mathrm{~g}$ de polpa, portanto compatível aos $113,62 \mathrm{mg} / 100 \mathrm{~g}$ de polpa apresentados para o caqui (CAVALCANTE et al., 2007b), e acima dos valores obtidos para limão e uva (LEE; KADER, 2000) com médias de $50,40 \mathrm{mg} / 100 \mathrm{~g}$ e 21,3 $\mathrm{mg} / 100 \mathrm{~g}$ de polpa, respectivamente. Por outro lado, os resultados do presente trabalho são inferiores à média reportada por Cavalcante et al. (2007a) para a acerola, que registraram resultados entre 575,48 e $1141,21 \mathrm{mg} / 100 \mathrm{~g}$ de polpa. De uma forma geral os valores contidos na Tabela 2 são considerados altos para uma fruta cítrica, considerando-se o nível mínimo adequado é de $50 \mathrm{mg} / 100 \mathrm{~g}$ de polpa (KIMBALL, 1991). Para a laranja, especificamente, Lee e Kader (2000) reportaram 75 e 54,7 mg/100g de polpa, respectivamente, para laranjas produzidas na Califórnia e na Flórida.

Em relação ao $\mathrm{pH}$, a laranja-da-terra apresentou media de 5,33, portanto inferior ao apresentado para o mamão (MESQUITA et al., 2007) com media de 5,4 , porém superior ao reportado por Damasceno Júnior e Bezerra (2002) para o caju, com média de 4,4. A acidez constitui fator de grande importância para o sabor e aroma dos frutos pois essa característica influencia o escurecimento oxidativo dos tecidos vegetais. A diminuição do seu valor acarreta redução da velocidade de escurecimento do fruto (BRAVERMAN, 1967).

\section{Conclusões}

Os resultados desse estudo indicam que a laranjada-terra tem boas qualidades físico-químicas. A média de $125,76 \mathrm{mg} / 100 \mathrm{~g}$ de fruta fresca indica que a laranja-da-terra é uma fonte natural de vitamina $\mathrm{C}$, com acidez baixa e elevado sólidos solúveis, com potencial para consumo in natura. Futuros estudos são necessários para determinar os parâmetros tecnológicos para o consumo da fruta da laranja-daterra fresca ou processada.

\section{Referências}

AWAD, M. Fisiologia pós colheita de frutos. São Paulo: Nobel, 1993. 114 p.

BRAVERMAN, J. B. S. Introducción a la bioquimica de los alimentos. Barcelona: Omega, 1967. cap. 14. 355 p.

CAVALCANTE, Í. H. L.; BECKMANN, M. Z.; MARTINS, A. B. G.; CAMPOS, M. C. C. Preliminary selection of acerola genotypes in Brazil. Fruits, Paris, v. 62 , n. 1, p. 1-8, 2007a. 
CAVALCANTE, Í. H. L.; MARTINS, A. B. G.; STUCHI, E. S. Fruit characteristics of eighteen orange cultivars. Revista de Biologia e Ciências da Terra, Campina Grande, v. 6, n. 2, p. 72-77, 2006.

CAVALCANTE, Í. H. L.; MARTINS, A. B. G; OLIVEIRA, I. V. M.; BECKMANN-CAVALCANTE, M. Z. Características de frutos de cinco variedades de caqui madurados en la planta o en post cosecha. Revista de Biologia e Ciências da Terra, Campina Grande, v. 7, n. 2, p. 201-209, 2007b.

CAVALCANTE, Í. H. L.; OLIVEIRA, I. V. M.; BECKMANN-CAVALCANTE, M. Z.; MARTINS, A. B. G. Substrate for seedling emergence of white sapote in Brazil. The Americas Journal of Plant Science and Biotechnology, London, v. 2, n. 2, p. 47-50, 2008.

CHITARRA, M. I. F.; CHITARRA, A. B. Pós-colheita de frutos e hortaliças: fisiologia e manuseio. Lavras: ESAL/ FAEPE, 1990. $320 \mathrm{p}$.

DAMASCENO JUNIOR, J. A.; BEZERRA, F. C. Qualidade de pedúnculo de cajueiro-anão precoce cultivado sob irrigação e submetido a diferentes sistemas de condução e espaçamento. Revista Brasileira de Fruticultura, Jaboticabal, v. 24, n. 1, p. 258-262, abr. 2002.

DONADIO, L. C.; FIGUEIREDO, J. O.; PIO, R. M. Variedades citricas. Jaboticabal: Funep, 1995. 297 p.

FOOD AND AGRICULTURE ORGANIZATION OF THE UNITED NATIONS - FAO. FAOSTAT: statistics database. 2008. Disponível em: <http://apps.fao.org/>. Acesso em: 10 Jun. 2008.

GOMES, P. Fruticultura brasileira. São Paulo: Nobel, 2007. $446 \mathrm{p}$.

KAYS, S. J. Preharvest factors affecting appearance. Postharvest Biology and Technology, Amsterdã, v. 15, n. 3, p. 233-247, 1999.

KIMBALL, D. A. Citrus processing: quality control e technology. AVI. New York: Van Nostrand Reinhold, $1991.473 \mathrm{p}$.

KLUGE, R. A.; NACHTIGAL, J. C.; FACHINELLO, J. C.; BILHALVA, A. B. Fisiologia e manejo pós-colheita de frutas de clima temperado. 2. ed. [S.1.]: Rural, 2002. $214 \mathrm{p}$.

LARA, A. B. W.; NAZÁRIO, G.; ALMEIDA, M. E. W.; PREGNOLATO, W. Normas analiticas do Instituto Adolfo Lutz. Métodos fisicos e químicos para análise de alimentos. 2. ed. São Paulo: Instituto Adolfo Lutz, 1976, v. $1,430 \mathrm{p}$.
LEE, S. K.; KADER, A. A. Preharvest and postharvest factors influencing Vitamin $\mathrm{C}$ content of horticultural crops. Postharvest Biology and Technology, Amsterdã, v. 20, n. 3, p. 207-220, 2000.

LORENZI, H.; BACHER, L.; LACERDA, M.; SARTORI, S. Frutas brasileiras e exóticas cultivadas. São Paulo: Instituto Plantarum, 2006. 640 p.

MARCHI, R. J. Modelagem de curvas de maturacao da laranja "Pêra" (citrus cinensis L. Osbeck) na região de Bebedouro-SP. Jaboticabal, 1993. Dissertação (Mestrado em Produção Vegetal) - Faculdade de Ciências Agrárias e Veterinárias, Universidade Estadual Paulista, São Paulo.

MATTHEIS, J. P.; FELLMAN, J. K. Preharvest factors influencing flavor of fresh fruit and vegetables. Postharvest Biology and Technology, Amsterdã, v. 15, $\mathrm{n}$. 3, p. 227-232, 1999.

MESQUITA, E. F. de; CAVALCANTE, L. F.; GONDIM, S. C. J.; CAVALCANTE, Í. H. L.; ARAÚJO, F. A. R. de; BECKMANN-CAVALCANTE, M. Z. Produtividade e qualidade de frutos do mamoeiro em função de tipos e doses de biofertilizantes. Semina: Ciências Agrárias, Londrina, v. 28, n. 3, p. 349-353, jul./set. 2007.

SILVA, F. de A. S. E; AZEVEDO, C. A. V. de. A new version of the assistat-statistical assistance software. In: WORLD CONGRESS ON COMPUTERS IN AGRICULTURE, 4., 2006, Orlando-FL-USA. Anais... Orlando: American Society of Agricultural Engineers, 2006. p. 393-396.

SOARES FILHO, W. S.; MEDRADO, A. C. M.; CUNHA, M. A. P.; CUNHA SOBRINHO, A. P.; PASSOS, O. S. Freqüência de híbridos em cruzamentos controlados de citros: cultivo de sementes versus cultivo in vitro de embriões. Pesquisa Agropecuária Brasileira, Brasília, v. 37, n. 7, p. 981-988, 2002.

STEGER, E. Trinta anos de desenvolvimento em processamento de citros, histórico, estado da arte e visão geral. Laranja, Cordeirópolis, v. 11, n. 2, p. 463-502. 1990.

VIANA, T. V. A.; VASCONCELOS, D. V.; AZEVEDO, B. M.; SOUZA, V. F. Estudo da aptidão agroclimática do Estado do Piauí para o cultivo da aceroleira. Ciência Agronômica, Fortaleza, v. 33, n. 2, p. 5-12, 2002.

VIÉGAS, F. C. P. A industrialização dos produtos cítricos. In: RODRIGUEZ, O.; VIÉGAS, F.; POMPEU JÚNIOR, J.; AMARO, A. A. (Ed.). Citricultura brasileira. 2. ed. Campinas: Fundação Cargill, 1991. v. 2, p. 898- 921. 